\title{
Peculiar Mechanism of Solubilization of a Sparingly Water Soluble Drug into Polymeric Micelles. Kinetic and Equilibrium Studies
}

\author{
Maria Liria Turco Liveri, ${ }^{* \dagger}$ Mariano Licciardi, ${ }^{\ddagger}$ Luciana Sciascia, ${ }^{\S}$ Gaetano Giammona, \\ and Gennara Cavallaro
${ }^{\dagger}$ Dipartimento di Chimica "Stanislao Cannizzaro”, University of Palermo, Viale delle Scienze Ed. 17, IT 90128, Palermo, Italy
${ }^{\ddagger}$ Dipartimento di Scienze e Tecnologie Molecolari e Biomolecolari (STEMBIO), University of Palermo, Via Archirafi 32, IT 90123, Palermo, Italy
${ }^{\S}$ Dipartimento di Scienze della Terra e del Mare (DiSTeM), University of Palermo, Via Archirafi 36, IT 90123, Palermo, Italy

\begin{abstract}
Complementary kinetic and equilibrium studies on the solubilization process of the sparingly water soluble tamoxifen (TAM) drug in polymeric aqueous solutions have been performed by using the spectrophotometric method. In particular, the amphiphilic copolymers obtained by derivatization of polymeric chain of poly(N-2-hydroxyethyl)-DLaspartamide, PHEA, with poly(ethylene glycol)s, PEG (2000 or $5000 \mathrm{Da})$, and/or hexadecylamine chain, $\mathrm{C}_{16}$, namely PHEA-PEG ${ }_{2000}-C_{16}$, PHEA-PEG ${ }_{5000}-C_{16}$, PHEA-C ${ }_{16}$, have been employed. Preliminary to the kinetic and equilibrium data quantitative treatment, the molar absorption coefficient of TAM in polymeric micelle aqueous solution has been determined. By these studies the solubization sites of TAM

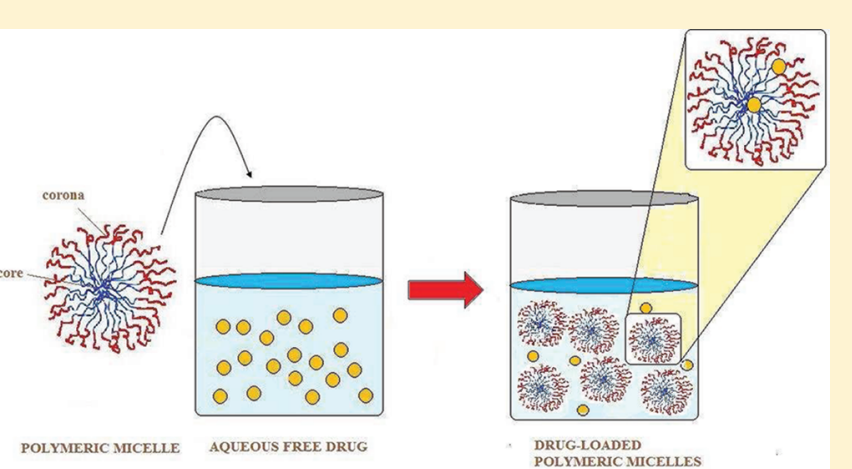
into the polymeric micelles have been determined and the solubilization mechanism has been elucidated through a nonconventional approach by considering the TAM partitioned between three pseudophases, i.e., the aqueous pseudophase, the hydrophilic corona, and the hydrophobic core. The simultaneous solution of the rate laws associated with each step of the proposed mechanism allowed the calculation of the rate constants associated with the involved processes, the values of which are independent of both the copolymer concentration and nature, with the exception of the rate of the TAM transfer from the corona to the core. This has been attributed to the steric barrier, represented by the corona, which hampers the solubilization into the core. The binding constant values of the TAM to the hydrophilic corona of the polymeric micelles, calculated through the quantitative analysis of the equilibrium data, depend on the thickness of the hydrophilic headgroup, while those of the hydrophobic core are almost independent of the copolymer type. Further confirmation to the proposed solubilization mechanism has been provided by performing the kinetic and equilibrium measurements in the presence of PHEA-PEG 2000 and PHEA-
\end{abstract} PEG $_{5000}$ copolymers.

\section{INTRODUCTION}

Polymeric micelles are nanosized systems obtained by the selfassembling at the proper concentration (critical aggregation concentration, cac) of amphiphilic polymeric unimers, i.e., polymers bearing both hydrophobic and hydrophilic chains; the obtained aggregates are characterized by a hydrophobic interior and a hydrophilic periphery, the so-called core and corona (palisade layer), respectively. Different kinds of molecules can be physically incorporated in the core or chemically linked to functional groups of the unimers and, depending on their hydrophobic/hydrophilic character, to be prevalent in the micelle core, on the surface, or in the palisade region. ${ }^{1}$

Polymeric micelles are emerging ${ }^{2-5}$ as promising drugdelivery vehicles because of their multimodular structure enabling them to actively target discrete cells, pass through biological barriers, and simultaneously carry drugs of various chemical nature into specific sites and within well-defined intervals of time.
Their wide application depends on some properties, such as the following: nanoscaled dimensions, able to allow their direct interaction with cell components of the ill tissue at the molecular level; ability to incorporate high amount of active molecules (high drug loading capacity) with subsequent increase of the efficiency of the drug delivery systems; welldefined and narrow size distribution that allows to obtain standardized drug release amount and rate; ability to deliver an intact form of chemically or physically unstable drugs, by increasing their bioavailability and decreasing administered doses, or making possible their administration, such as, for example, for nucleic acid based drugs. Moreover, it is possible to obtain an efficient localization of the drug in the target site, through the use of targeting portions, properly anchored on the

Received: December 12, 2011

Revised: March 1, 2012

Published: March 30, 2012 
surface of the nanosystems and characterized by high cell specificity.

For several years the ability to act as parenteral drug delivery systems of poly(hydroxyethylaspartamide) (PHEA) copolymer based micelles have been investigated in our laboratory. PHEA is a synthetic polymer having protein-like structure, obtained by the reaction of ethanolamine with poly(succinimide) (PSI), itself prepared by thermal polycondensation of D,L-aspartic acid. ${ }^{6}$ PHEA has good biopharmaceutical properties ${ }^{7-11}$ as drug carrier material such as high water solubility, multifunctionality, biocompatibility, and low cost of production.

In a previous paper, poly(hydroxyethylaspartamide) (PHEA) derivatives bearing poly(ethylene glycol) (2000 or $5000 \mathrm{Da})$ or both poly(ethylene glycol) and hexadecylalkylamine $\left(\mathrm{C}_{16}\right)$ as pendant moieties were investigated as polymeric colloidal drug carriers. The ability of the PHEA derivatives to solubilize hydrophobic drugs was investigated using paclitaxel, amphotericin $\mathrm{B}$, and methotrexate ${ }^{9}$ or dexamethasone. ${ }^{11}$ The results demonstrated that the drug solubility and delivery depend on both macromolecule composition and drug physicochemical properties. In particular, PEG/hexadecylalkylamine cografting increased significantly the solubilization properties of PHEA for the considered drugs while the conjugation of PEG only did not endow PHEA with drug carrier properties.

However, in any case, the application of polymeric micelles as drug delivery systems of sparingly soluble substances cannot leave out of consideration the wide and in-depth study of the drug-water solubilization. To date, most of the studies have dealt with aspects of solubilization under equilibrium conditions $^{12-19}$ whereas little attention, because of appreciable experimental difficulties, has been paid to the kinetics of the solubilization process. A knowledge of the kinetics of the solubilization process is very important particularly in areas such as drug delivery and carrying. In fact, knowing the temporal scale of the process is often more important than identifying the final equilibrium state of the system. In other words, not only is the total amount of solute important, determined by the thermodynamic equilibrium state of the system, but also the rate of the system composition change.

For this reason, a systematic spectrophotometric study of the kinetic of the solubilization process of the drug has been performed in our laboratory, focusing our attention on poly(aspartamide) copolymers as unimers forming polymeric micelles and considering tamoxifen as sparingly water soluble model drug.

As copolymers we have used the series PHEA-PEG ${ }_{2000}-C_{16}$, PHEA-PEG ${ }_{5000}-\mathrm{C}_{16}$, and PHEA-C $\mathrm{C}_{16}$ having different hydrophilic/hydrophobic ratios, whose physicochemical characterization has been previously performed. ${ }^{20}$ Briefly, it has been obtained that these copolymers are able to self-aggregate into micelles and the cac is very low. In the present study, the measurements have been carried out at copolymer concentrations higher than the cac previously determined.

Since each solubilization process reaches the equilibrium state, the final absorbance value has been recorded, for all copolymer concentrations, with the aim of determining the molar solubilization capacity $(\chi)$ and the binding constant $\left(K_{\mathrm{B}}\right)$ of the TAM to the aggregated systems. ${ }^{12-16}$ However, since this kind of analysis did not yield reliable results, both the kinetic and the equilibrium data have been treated with a nonconventional approach. This way, it was possible to elucidate the solubilization mechanism also in the light of determination of the molar absorption coefficient of TAM in polymeric micelle aqueous solution. Further confirmation of the proposed mechanism has been provided by performing the solubilization measurements in the presence of PHEA-PEG ${ }_{2000}$ and PHEA-PEG 5000 copolymers.

\section{EXPERIMENTAL METHODS}

Materials. Tamoxifen (TAM) and decane were furnished from Aldrich Chemical Co. and used without any further purification. $O$-(2-aminoethyl)-O'-methylpoly(ethylene glycol) $2000\left(\mathrm{PEG}_{2000}-\mathrm{NH}_{2}\right) \quad(<0.4 \mathrm{mmol} \mathrm{NH} / \mathrm{g})$ and $\mathrm{O}$-(2-aminoethyl)-O'-methyl(polyethylene glycol) $5000\left(\mathrm{PEG}_{5000}-\mathrm{NH}_{2}\right)$ $\left(<0.17 \mathrm{mmol} \mathrm{NH}_{2} / \mathrm{g}\right)$ and all the other chemicals were purchased from Fluka. $\alpha, \beta$-Poly $(N$-2-hydroxyethyl)-DL-aspartamide (PHEA) and the PHEA derivatives (PHEA-PEG ${ }_{2000}$, PHEA-PEG ${ }_{5000}$, PHEA-PEG ${ }_{2000}-\mathrm{C}_{16}$, PHEA-PEG ${ }_{5000}-\mathrm{C}_{16}$, and PHEA-C $\mathrm{C}_{16}$ ) were prepared and purified according to a

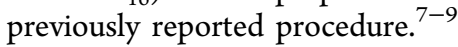

Stock solutions of all copolymers were prepared by weight. All solutions were vigorously shaken several times and equilibrated 1 day at room temperature before use.

All solutions were prepared by using deionized water, having a resistivity higher than $1 \mathrm{M} \Omega \mathrm{cm}$, obtained from reverse osmosis (Elga, model Option 3).

Spectrophotometric Measurements. Kinetic measurements and UV-vis spectra were performed with a computercontrolled Beckman spectrophotometer (model DU-640), equipped with six thermostated compartments for $1.00 \mathrm{~cm}$ cuvettes and appropriate magnetic stirring devices. The temperature control was obtained by a thermostat Heto Therm (Thermo Karlsruhe, Germany) $t \pm 0.1{ }^{\circ} \mathrm{C}$.

The mixtures for the kinetic runs were prepared directly in the cuvettes by adding $3 \mathrm{~mL}$ of aqueous copolymer solutions, at the desired concentrations, to $4 \times 10^{-4} \mathrm{~g}$ of TAM. It is worth noting that the amount of TAM was always higher with respect to its solubility in water $\left(0.6 \mu \mathrm{g} \mathrm{mL}^{-1}\right)$.

The copolymers' concentrations were varied over wide concentration ranges.

The progress of the TAM solubilization was followed both in the absence and presence of copolymers under constant stirred conditions by monitoring the UV spectra time evolution. Then, the wavelength of $275 \mathrm{~nm}$ was selected and the maximum absorbance increase was evidenced. At the end of the process, the obtained suspensions were filtered through $0.45 \mathrm{~mm}$ cellulose membrane filters, and the spectra of the final solutions were recorded.

The solubilization kinetic data both in the absence and presence of copolymers have been analyzed by applying several models and the discrimination among the different rate laws has been done by means of various statistics ${ }^{21}$ such as ANOVA $F$ Test, PRESS, AIC, $\mathrm{R}, \mathrm{R}^{2}$, and the analysis of the residuals. It has been obtained that the absorbance vs time profile did not fit first-order or second-order rate laws, suggesting a multistep pathway. Moreover, it has been noticed that the experiments both in the absence and presence of copolymers were reproducible only when the samples were not placed in the first position of the spectrophotometer. This suggests a certain role played by the time of exposure to the UV radiation during the course of the experiment, which in turn depends on the position of the cuvettes within the spectrophotometer. More precisely, we have to take into account that the first position of the instrument is continuously subjected to the UV radiation, while in the other ones the samples are irradiated only for the time required for the measurements. In order to discern 
between the above-proposed possibilities, we have studied the kinetics of TAM solubilization in pure water and in aqueous copolymer solutions, under the same experimental conditions reported above, as a function of the position of the cuvettes within the spectrophotometer. It was found that the process of solubilization in water follows first-order kinetics when we placed the cuvettes in the last five positions of the spectrophotometer, while the rate law is more complex when the sample was placed in the first position. Moreover, independent of the position in the spectrophotometer where the solubilization process takes place in the presence of copolymers, the rate law is complicated. The results in pure water have been interpreted by taking into account that UV light induces a transition ${ }^{22}$ from the trans to the cis isomer of the TAM; as a consequence, the coexistence of two different species implies that they solubilize with different rate laws.

The presence of the two isomers has been checked by performing HPLC analysis of the TAM aqueous filtered solutions, obtained both in the absence and in the presence of copolymers. It was found that the chromatogram of the solutions prepared under constant UV radiation exposure presents two different peaks which have been attributed ${ }^{22}$ to the trans and the cis isomers, while that of the samples exposed to the radiation just for the time required for a measurement presents only the peak corresponding to the trans isomer. In the light of these results, we decided to carry out all the measurements by using the last five positions of the spectrophotometer.

Since the HPLC analysis indicated only one TAM isomer in the aqueous copolymer solutions, we have ruled out that the complicated rate law is due to two isomers, and we have proposed multistep pathways, the mechanism of which has been simulated by Gepasi modeling. The fits were reproducible to within $\pm 3 \%$.

Triplicate experiments were performed for both the aqueous solution and each copolymer concentration. The copolymer concentrations were kept the same in the reference and measurement cells to eliminate the effect of copolymer on UV absorbance.

The UV spectra of the TAM aqueous solution and TAM copolymer acqueous solution, for all the concentrations used in the present work, have also been monitored as a function of TAM concentration to determine the molar absorption coefficient in both pure water and biopolymer solutions. For comparison, the spectrophotometric measurements have been carried out by using two organic solvents, i.e., methanol and decane.

\section{RESULTS AND DISCUSSION}

The kinetic of the solubilization of TAM in the polymeric aqueous solutions has been investigated over a wide range of polymers concentration, always higher than CAC previously determined, ${ }^{19}$ by using the spectrophotometric method. As copolymers, we have used the series PHEA-PEG $\mathrm{P}_{2000}-\mathrm{C}_{16}$, PHEA-PEG ${ }_{5000}-\mathrm{C}_{16}$, and PHEA-C $\mathrm{C}_{16}$ having different hydrophilic/hydrophobic ratios. Since each solubilization process reaches the equilibrium state, the final absorbance value has been recorded for all copolymer concentrations.

For the sake of clarity, we have divided the Results and Discussion into subsections. The quantitative analysis of the kinetic data was performed by taking into account the important results obtained from the determination of the molar absorption coefficient of TAM in copolymer aqueous solutions. This allowed us not only to propose the solubilization mechanism but also to confirm the solubilization sites of the TAM into the polymeric micelles. Further confirmation of both the proposed mechanism and solubilization sites has been provided by performing the solubilization measurements in the presence of PHEA-PEG 2000 and PHEA$\mathrm{PEG}_{5000}$ copolymers. In the light of the kinetic results, the equilibrium data have been treated with a nonconventional approach, and the quantitative treatment yielded the binding constant values of the TAM to the hydrophilic corona and hydrophobic core of the polymeric micelles. This way, the complementary equilibrium and kinetic data support each other.

3.1. Solubilization Kinetic Data. The time dependence of the maximum absorbance increase obtained at the selected wavelength for the TAM solubilization in pure water is shown in Figure 1A, while a typical absorbance profile obtained for the

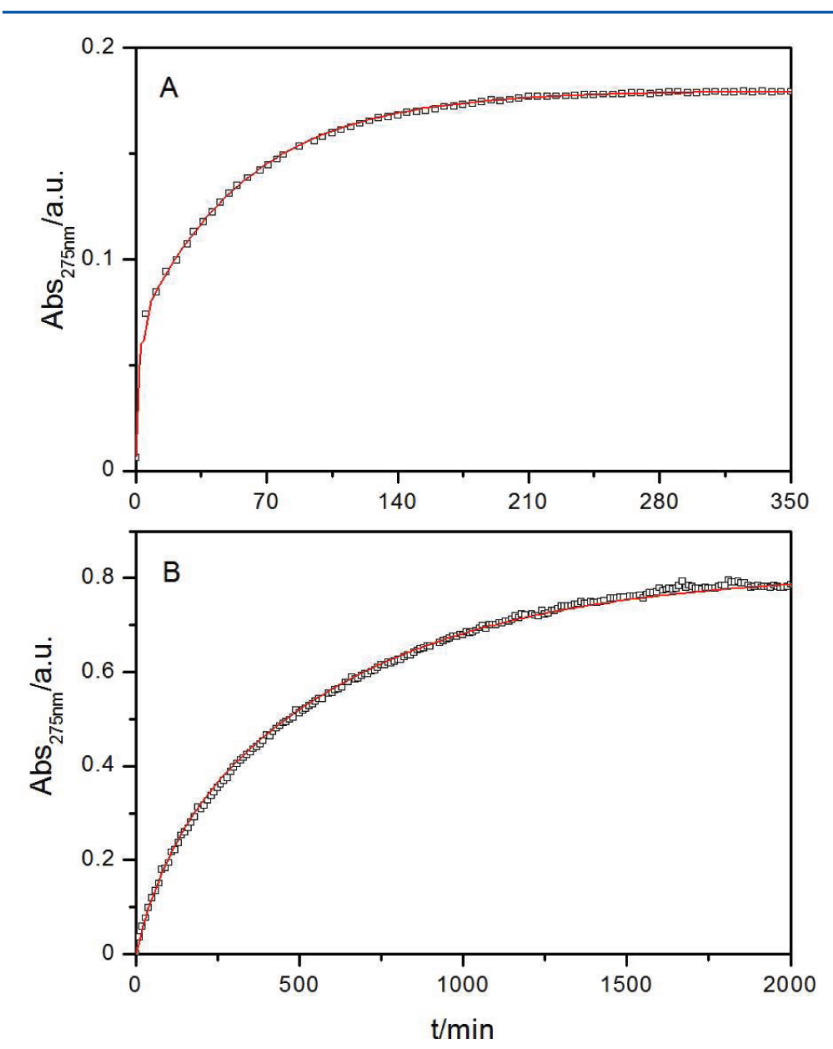

Figure 1. Time course of the TAM solubilization in pure water (A) and in the presence of $1.4 \mathrm{~g} \mathrm{dm}^{-3}$ of PHEA-PEG ${ }_{5000}-\mathrm{C}_{16}(\mathrm{~B}), \mathrm{TAM}=$ $4 \times 10^{-4} \mathrm{~g}, t=25.0{ }^{\circ} \mathrm{C}$.

TAM solubilization in the presence of the polymeric micelles is depicted in Figure 1B.

It is worth mentioning that, for all the copolymer concentrations investigated and type of copolymers, the time course of the solubilization of TAM looks different from that obtained in pure water. In particular, the solubilization process in the presence of micelles needs more time to reach the equilibrium state and depends on both the concentration of polymer and its nature. Moreover, the final absorbance values are always higher than those recorded in pure water. This implies that the polymeric micelles are able to solubilize a larger extent of the TAM drug.

It is well-known that one of the main difficulties concerning the analysis of the kinetic data is to identify the correct rate laws 
and to propose the mechanism. As for the solubilization of sparingly water soluble molecules in both aqueous and micellar solution, either a simple first-order saturation equation or a pseudo-second-order model is used, ${ }^{23,24}$ whose equations are respectively

$$
\begin{aligned}
& \frac{\mathrm{d} C}{\mathrm{~d} t}=k_{1}\left(K C^{*}-C\right) \\
& \frac{\mathrm{d} C}{\mathrm{~d} t}=k_{2}\left(K C^{*}-C\right)^{2}
\end{aligned}
$$

where $C^{*}$ is the saturation concentration of the drug (mol $\mathrm{dm}^{-3}$ ), $C$ the time variable concentration of the drug (mol $\left.\mathrm{dm}^{-3}\right), k_{1}$ is the first-order rate constant $\left(\mathrm{min}^{-1}\right), k_{2}$ is the second-order rate constant $\left(\mathrm{dm}^{3} \mathrm{~mol}^{-1} \mathrm{~min}^{-1}\right)$, and $K$ is the aqueous solubility enhancement factor due to the presence of surfactants. Note that eqs 1 and 2 in the case of solubilization in water do not contain the $K$ factor.

Integration of the above equation leads to

$$
\begin{aligned}
& C=K C^{*}\left(1-\mathrm{e}^{-k_{1} t}\right) \\
& C=K C^{*} \frac{K C^{*} k_{2} t}{1+K C^{*} k_{2} t}
\end{aligned}
$$

for the first- and the second-order models, respectively.

In order to analyze the kinetic data, it is necessary to convert the absorbance data into concentration ones. For this purpose, we have, according to the procedure reported in the literature, ${ }^{13-15}$ determined the molar absorption coefficient of the TAM both in aqueous and in methanolic solution.

The various statistics show that for the TAM solubilization in pure water the first-order rate law is fulfilled, and the fitting of the experimental data yields the kinetic constant value as $k_{\mathrm{w}}=$ $0.015 \mathrm{~min}^{-1}$, while in the presence of copolymers the abovereported models resulted to be inadequate. We have ascribed the failure to the inadequacy of the use of the molar adsorption coefficient determined in methanolic solution. Thus, we have determined the molar adsorption coefficients of TAM in aqueous polymeric micelle solutions, whose results are reported in the following subsection.

3.1a. Molar Absorption Coefficients Determination. The molar absorption coefficients of TAM at $275 \mathrm{~nm}$ in pure water and methanol and in aqueous polymer solutions were determined by monitoring the UV spectra of the solutions at various TAM concentrations. Typical calibration curves for the different experimental conditions used are depicted in Figures 2 and 3. It has been found that in pure water and methanol and in the presence of the copolymers of PHEA-PEG the absorbance increases linearly with the TAM concentration; i.e., the Lambert-Beer law is fulfilled. This way, the linear least-squares analysis of the absorbance data allows us to estimate the molar absorption coefficient of TAM in methanol $\left(\varepsilon_{\mathrm{MeOH}}=(8.2 \pm\right.$ $\left.0.1) \times 10^{3} \mathrm{dm}^{3} \mathrm{~cm}^{-1} \mathrm{~mol}^{-1}\right)$ and in pure water $\left(\varepsilon_{\mathrm{w}}=(1.1 \pm 0.1)\right.$ $\left.\times 10^{5} \mathrm{dm}^{3} \mathrm{~cm}^{-1} \mathrm{~mol}^{-1}\right)$ and in the presence of the copolymers of PHEA-PEG $\left(\varepsilon_{\mathrm{PP}}=(1.0 \pm 0.1) \times 10^{5} \mathrm{dm}^{3} \mathrm{~cm}^{-1} \mathrm{~mol}^{-1}\right)$. The invariance of the $\varepsilon$ determined in the PHEA-PEG with respect to that calculated in pure water suggested that the TAM in the PHEA-PEG solutions experiences an environment akin to pure water; i.e., in the random coil it has to be located in the hydrophilic region.

On the contrary, a deviation from linearity is brought about by the presence of the $\mathrm{C}_{16}$ moiety in the polymers, which
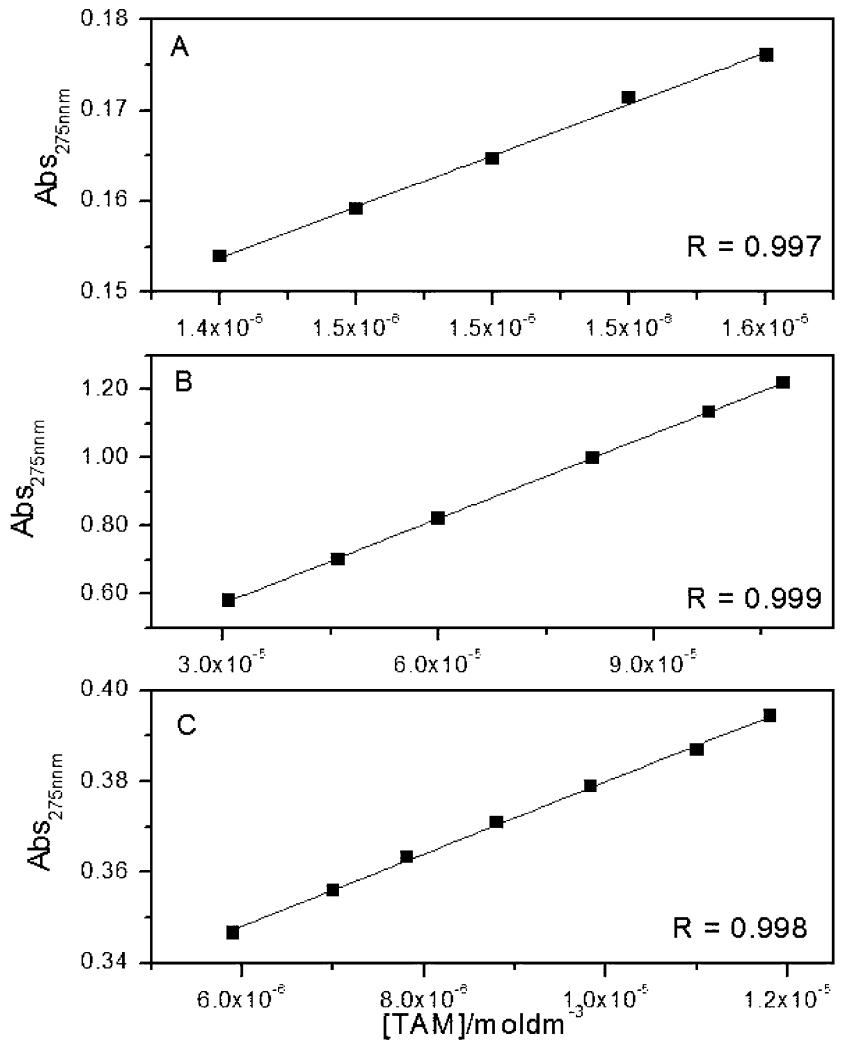

Figure 2. Calibration curves of absorbance vs concentration for TAM at $275 \mathrm{~nm}$ in (A) pure water, (B) methanol, and (C) decane.

clearly indicates that the total absorbance value is due to different contributions from $\varepsilon$ different values.

Moreover, it is worth saying that at any TAM concentration the spectrum in the presence of PHEA-PEG copolymers perfectly matches with that in pure water whereas the hydrophobic part $\mathrm{C}_{16}$ in the polymer backbone leads to a change in the shape (see Figure 4).

By taking into account that the hydrophobic core has the same properties of an organic solvent, we have monitored the UV spectra of the TAM-decane solutions. A typical spectra is shown in Figure 4. It has been obtained that the Lambert-Beer law is satisfied (see Figure 2C), allowing us to estimate the molar absorption coefficient $\left(\varepsilon_{2}=(8.0 \pm 0.1) \times 10^{3} \mathrm{dm}^{3} \mathrm{~cm}^{-1}\right.$ $\left.\mathrm{mol}^{-1}\right)$. This value is very close to that obtained in methanol.

It is worth saying that, for any PHEA-PEG- $\mathrm{C}_{16}$ copolymers used, the TAM copolymer aqueous solution spectrum is given by the sum of the spectrum of TAM in decane and that in the corresponding PHEA-PEG copolymer (see Figure 4 as typical spectra).

Moreover, perusal of Figure 4 also indicates that the spectrum of the TAM drug measured in the presence of PHEA-PEG- $\mathrm{C}_{16}$ copolymers wholly overlaps with that in methanol.

These results are a clear indication that the TAM has two solubilization loci, namely, the hydrophilic corona and the hydrophobic core of the micelle.

3.1b. Analysis of the Kinetic Data. In light of the molar adsorption results, we could reasonably modify the solubilization mechanism by considering that different regions of the polymeric micelles, namely the hydrophilic corona and the hydrophobic core, incorporate the TAM drug with different 


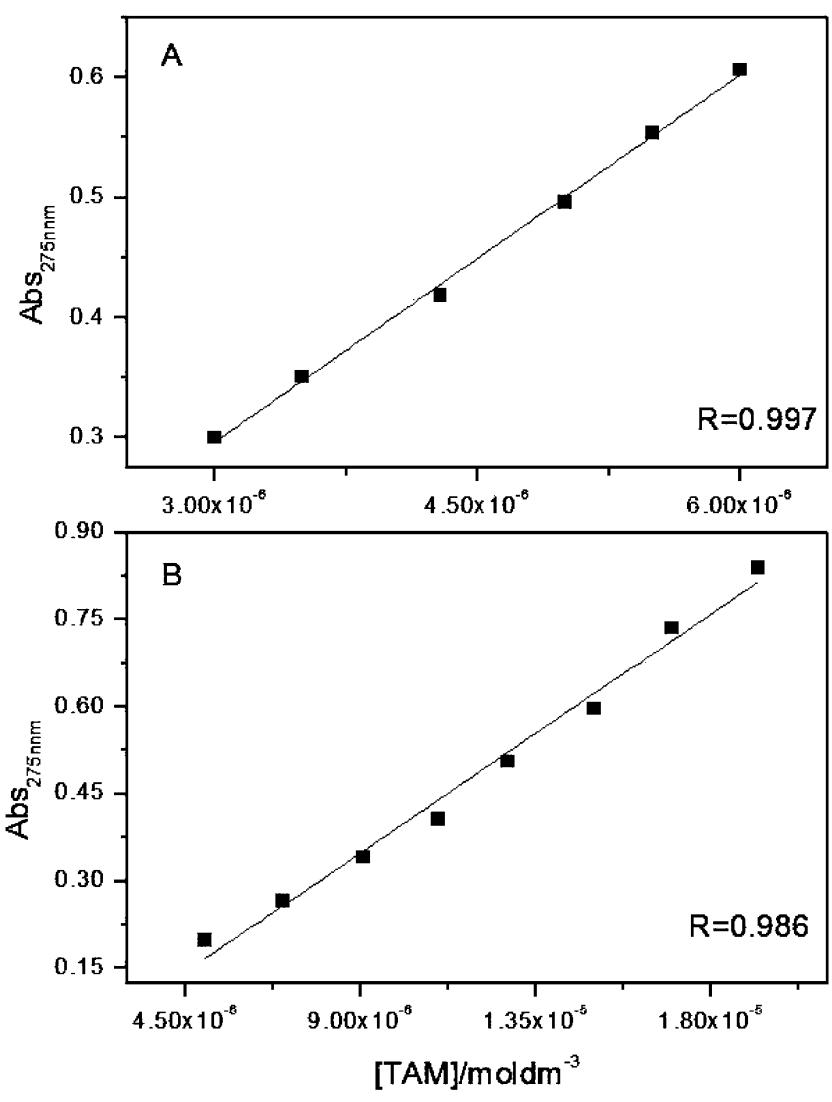

Figure 3. Calibration curves of absorbance vs concentration for TAM at $275 \mathrm{~nm}$ in (A) $1.4 \mathrm{~g} \mathrm{dm}^{-3}$ of PHEA-PEG 5000 and (B) $1.4 \mathrm{~g} \mathrm{dm}^{-3}$ of PHEA-PEG ${ }_{5000}-\mathrm{C}_{16}$.

rates. The solubilization mechanisms with the associated rate laws are given in Scheme 1.

The above-reported dissolution scheme implies that the solid TAM solubilizes in both water and the palisade layer ( $p$ ) according to reactions $\mathrm{R} 1$ and $\mathrm{R} 2$, respectively, then the

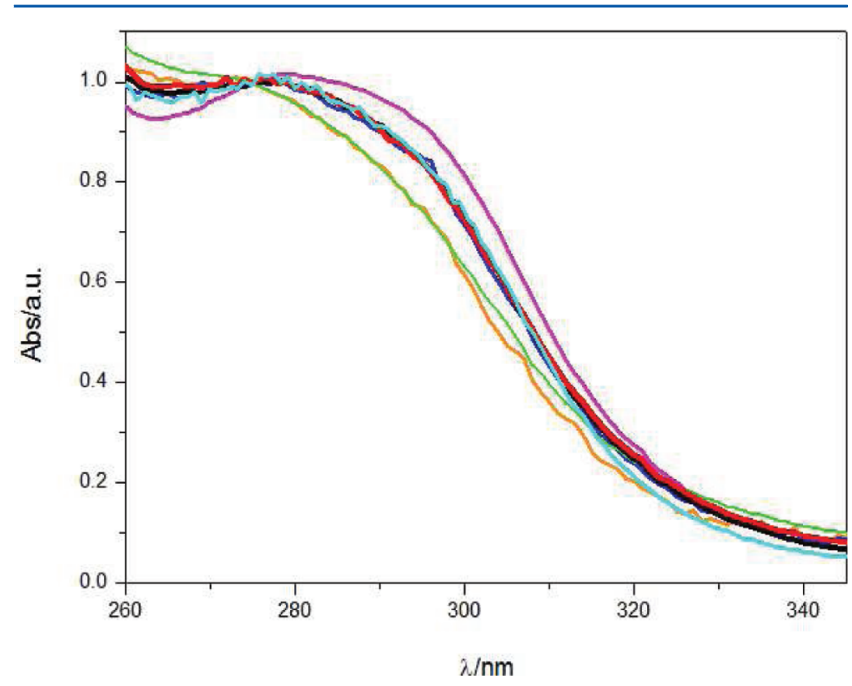

Figure 4. Normalized UV/vis spectra of TAM solubilized in methanol (cyan line), pure water (orange line), decane (magenta line), $1.4 \mathrm{~g}$ $\mathrm{dm}^{-3}$ of PHEA-PEG 2000 (green line), $1.4 \mathrm{~g} \mathrm{dm}^{-3}$ of PHEA-PEG $2000^{-}$

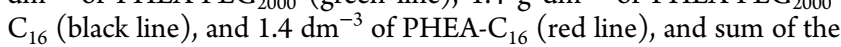
normalized UV/vis spectra for TAM solubilized in pure water and TAM solubilized in decane (blue line), $t=25.0{ }^{\circ} \mathrm{C}$. aqueous TAM is transferred to the hydrophilic corona from which it migrates to the hydrophobic core according to the equilibrium reactions $\mathrm{R} 3$ and $\mathrm{R} 4$, respectively.

The simultaneous solution of the rate laws associated with each step of the solubilization Scheme 1 allows us to estimate the kinetic constants, whose values are collected in Table 1. It has to be said that the kinetic constant values for all copolymer investigated are independent of the copolymer concentration; thus, the mean values are reported in Table 1 .

In order to corroborate the above-proposed mechanism and to establish the role played by the corona of the micelles, we have studied TAM solubilization in aqueous solution of PHEA$\mathrm{PEG}_{2000}$ and PHEA-PEG 5000 . It has been noted that, even though the PHEA-PEG copolymers do not self-associate, they are able to solubilize the TAM, most likely in the macromolecular coil. Typical absorbance profiles at the selected wavelength as a function of time are illustrated in Figure 5.

It can be easily seen that the shapes of these curves resemble those obtained in the presence of polymeric micelles and indeed, as various statistics demonstrate, the TAM dissolution does not follow either a simple first-order saturation equation or a pseudo-second-order model. Analogously to the polymeric micelles, we have proposed a multistep dissolution mechanism as in Scheme 2.

Analogously to the kinetic data obtained for the solubilization process in polymeric micelles, we have done the simultaneous solution of the rate laws associated with each step shown in Scheme 2. This allowed us to estimate the kinetic constants, whose values are collected in Table 1. Also in this case, the kinetic constant values for the two copolymers investigated are independent of the copolymer concentration; thus, the mean values are reported in Table 1.

Perusal of Table 1 underlines that the kinetic constant values for the TAM solubilization in water are in good agreement with those directly measured, indirectly confirming the appropriateness of the kinetic analysis. Moreover, for all copolymers used, the solubilization rate constant value of TAM in pure water is always higher than those obtained in the presence of polymers, which indeed are independent of the copolymer nature. This result could be explained by taking into account that, in the absence of copolymer, the water molecules are more available for the dissolution of the drug, while, in the copolymer solutions, they are attracted toward the hydrophilic corona of the aggregate, and as a consequence the solubilization process is delayed.

Slower dissolution rates of sparingly water soluble molecules in aqueous micellar solution than in water have also been previously $^{23,25}$ obtained.

As to the $k_{3}$ values, we can notice that they are independent of the thickness of the hydrophilic corona, but we can say that, once the TAM molecules penetrate the palisade layer, it leaves it $\left(k_{-3}\right)$ with a slow rate. On the contrary, the transfer of the TAM from the corona to the hydrophobic core of the micelles depends on the type of copolymer-forming micelles. In particular, the TAM goes through the corona to reach the core much faster when the palisade layer does not contain the PEG units. This indeed demonstrates that the hydrophilic corona represents a very steric barrier which slows down the solubilization into the core. Moreover, the release of the TAM from the core is independent of the copolymer nature. This result is in line with the consideration that the three copolymers used possess the same hydrophobic tail. 
Scheme 1

$$
\begin{aligned}
& \operatorname{TAM}_{(s)} \stackrel{k_{1}}{\longrightarrow} T A M_{(w)} \\
& v_{1}=k_{1}\left(C^{*}-[T A M]_{w}\right) \\
& \operatorname{TAM}_{(s)} \stackrel{k_{2}}{\longrightarrow} \operatorname{TAM}_{(P)} \\
& v_{2}=k_{2}\left(K C *-[T A M]_{p}\right) \\
& \text { R2 } \\
& T A M_{(w)} \underset{k-3}{\stackrel{k 3}{\rightleftarrows}} \operatorname{TAM}_{(P)} \\
& v_{3}=k_{3}[T A M]_{w}-k_{-3}[T A M]_{p}
\end{aligned}
$$

Table 1. Averaged Values for Rate Constants Associated with the Reactions in Scheme 1

\begin{tabular}{lccccc} 
& $k_{1} / \mathrm{min}^{-1}$ & $k_{2} / \mathrm{min}^{-1}$ & $k_{3} / \mathrm{min}^{-1}$ & $k_{-3} / \mathrm{min}^{-1}$ & $k_{4} / \mathrm{min}^{-1}$ \\
$\mathrm{H}_{2} \mathrm{O}$ & 0.015 & & & & \\
PHEA-C $_{16}$ & 0.015 & 0.0023 & $8.7 \times 10^{-4}$ & $1.9 \times 10^{-4}$ & $1.5 \times 10^{-3}$ \\
PHEA-PEG $_{2000}-\mathrm{C}_{16}$ & 0.014 & 0.0024 & $7.0 \times 10^{-4}$ & $2.0 \times 10^{-4}$ & $9.9 \times 10^{-4}$ \\
PHEA-PEG $_{5000}-\mathrm{C}_{16}$ & 0.015 & 0.0023 & $1.0 \times 10^{-3}$ & $2.0 \times 10^{-4}$ & $5.5 \times 10^{-4}$ \\
PHEA-PEG $_{2000}$ & 0.014 & 0.0022 & $9.1 \times 10^{-4}$ & $2.1 \times 10^{-4}$ & $1.4 \times 10^{-4}$ \\
PHEA-PEG $_{5000}$ & 0.016 & 0.0025 & $8.5 \times 10^{-4}$ & $2.0 \times 10^{-4}$ & \\
\hline
\end{tabular}

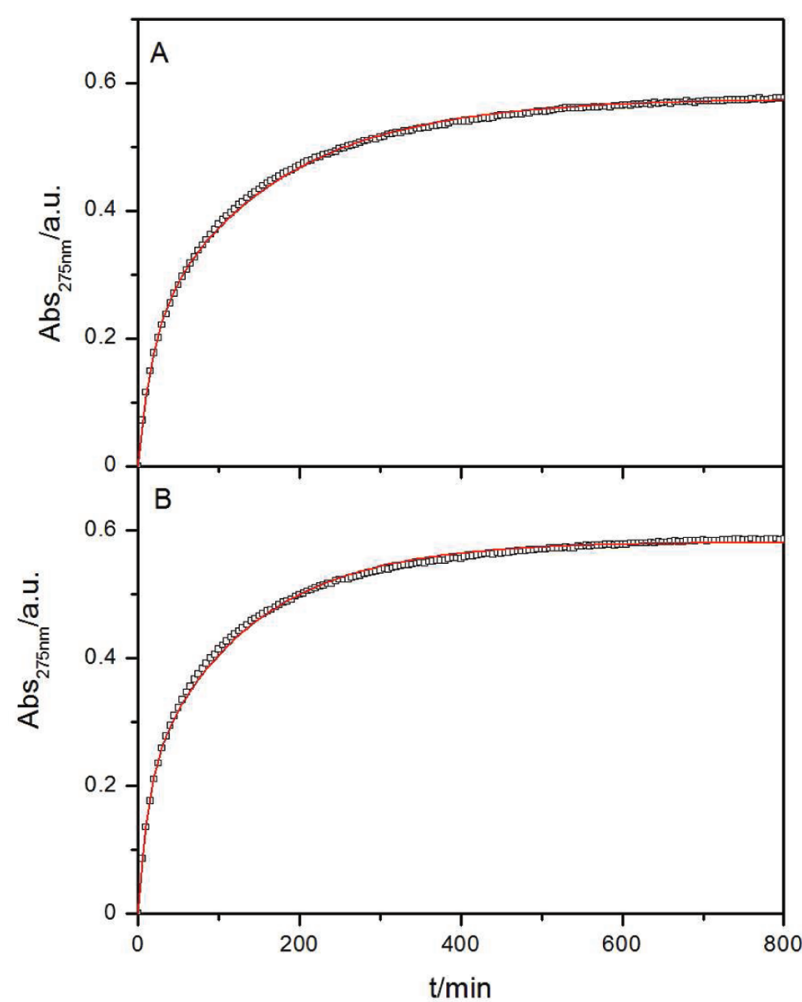

Figure 5. Time course of the TAM solubilization in the presence of $1.4 \mathrm{~g} \mathrm{dm}^{-3}$ of PHEA-PEG 2000 and $1.4 \mathrm{~g} \mathrm{dm}^{-3}$ of PHEA-PEG 5000 ; TAM $=4 \times 10^{-4} \mathrm{~g}, t=25.0^{\circ} \mathrm{C}$.

Moreover, the quantitative treatment of the kinetic data yields the values of the aqueous solubility enhancement factor $(K)$, whose plots as a function of polymer concentrations are depicted in Figures 6 and 7. As can be noticed for all the

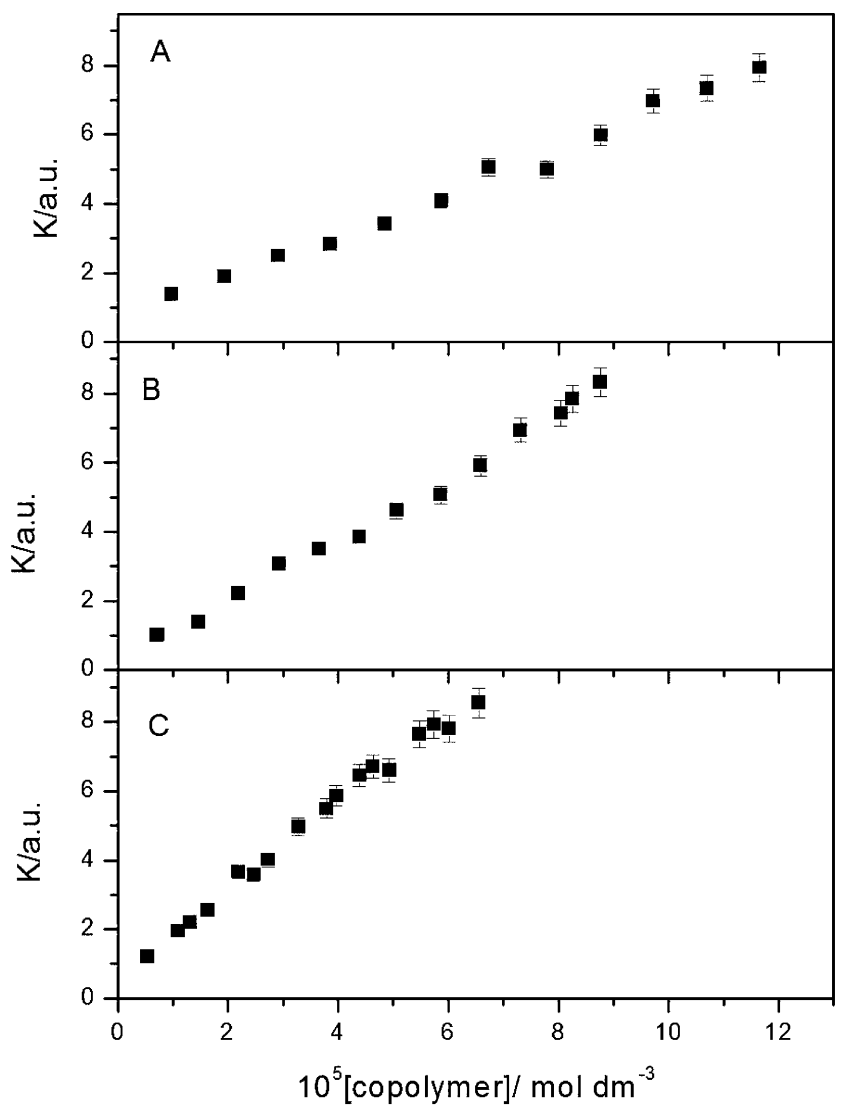

Figure 6. Plot of the aqueous solubility enhancement factor $(K)$ as a function of PHEA-C $\mathrm{C}_{16}$ (A), PHEA-PEG ${ }_{2000}-\mathrm{C}_{16}$ (B), and PHEA$\mathrm{PEG}_{5000^{-}} \mathrm{C}_{16}(\mathrm{C})$ concentrantions; $\mathrm{TAM}=4 \times 10^{-4} \mathrm{~g}, t=25.0^{\circ} \mathrm{C}$.

Scheme 2

$$
\begin{aligned}
& T A M_{(s)} \stackrel{k 1}{\longrightarrow} T A M_{(w)} \\
& v_{1}=k_{1}\left(C^{*}-[T A M]_{w}\right) \\
& \operatorname{TAM}_{(s)} \stackrel{k 2}{\longrightarrow} \operatorname{TAM}_{(P)} \\
& v_{2}=k_{2}\left(K C^{*}-[T A M]_{p}\right) \\
& \text { R2 } \\
& T A M_{(w)} \underset{k-3}{\stackrel{k 3}{\rightleftarrows}} \operatorname{TAM}_{(P)} \\
& v_{3}=k_{3}[T A M]_{w}-k_{-3}[T A M]_{p} \\
& \operatorname{TAM}_{(P)} \underset{k-4}{\stackrel{k 4}{\rightleftarrows}} \operatorname{TAM}_{(C)} \\
& v_{4}=k_{4}[\text { TAM }]_{p}-k_{-4}[\text { TAM }]_{c}
\end{aligned}
$$




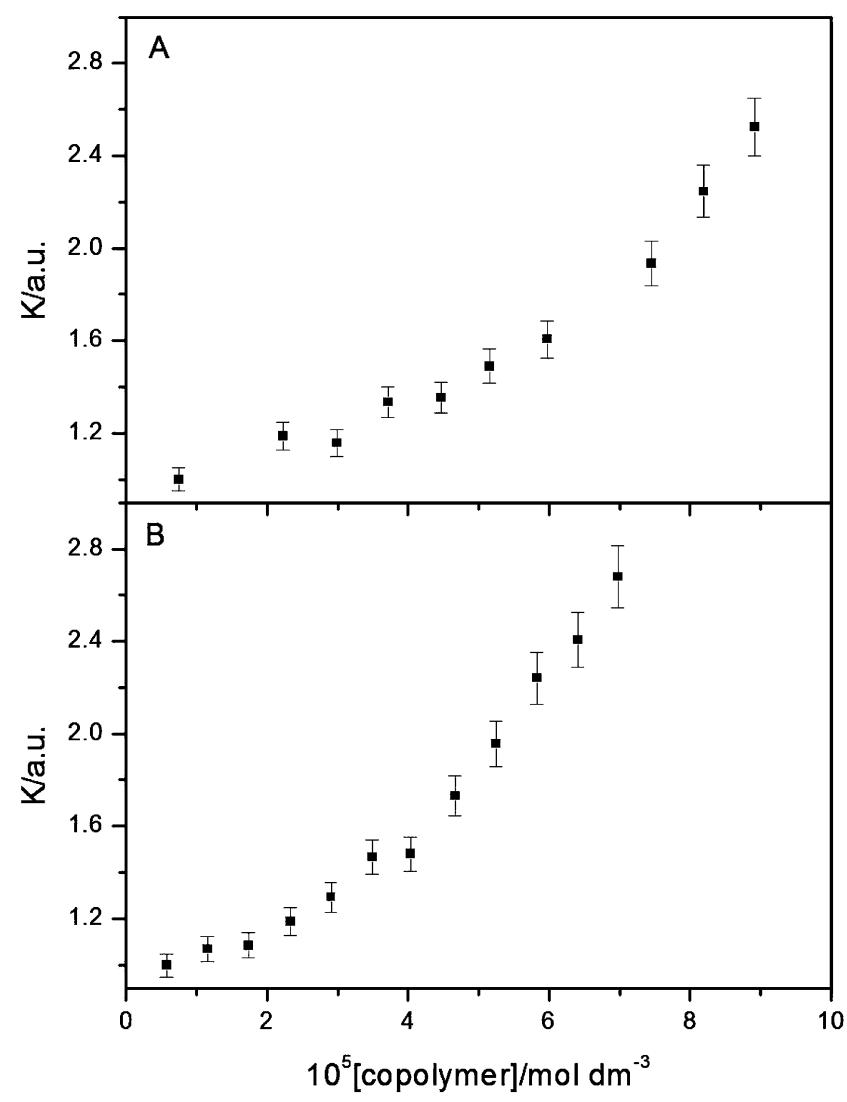

Figure 7. Plot of the aqueous solubility enhancement factor $(K)$ as a function of PHEA-PEG 2000 (A) and PHEA-PEG ${ }_{5000}(\mathrm{~B}) ; \mathrm{TAM}=4 \times$ $10^{-4} \mathrm{~g}, t=25.0^{\circ} \mathrm{C}$.

copolymers used, $K$ increases on increasing copolymer concentration and reaches a maximum value of about 8 when the polymer possesses the $\mathrm{C}_{16}$ hydrophobic tail, while only an enhancement factor about 3 is obtained for the PHEA-PEG copolymers.

Further evidence for the TAM solubilization sites into polymeric micelles is provided by the analysis of the TAM spectra evolution. In particular, as shown in a typical situation, Figure 8 , the TAM spectrum progressively changes as a function of time.

It can be noticed that at the beginning the shape of the spectrum is like that obtained in pure water and in the presence of PHEA-PEG copolymers while with passing time it shows the features of the spectrum in decane and that in the corresponding PHEA-PEG-C ${ }_{16}$ copolymer (see Figure 4). These findings not only give information on the solubilization sites of the TAM in the aggregated systems but also corroborate that hydrophilic corona of the nanostructured aggregate represents a barrier which hampers the direct transfer of drug from the aqueous to the hydrophobic region of the micellar phase.

3.2. Binding of TAM to Polymeric Aggregates. The final absorbance values, which indicate that the solubilization process has reached the equilibrium state obtained in the kinetic measurements, as a function of polymeric micelles concentration are depicted in Figure 9.

Usually, the absorbance data are converted into concentration of a species to determine two descriptors, ${ }^{13-16}$ i.e., the molar solubilization capacity $(\chi)$ and the binding constant $\left(K_{\mathrm{B}}\right)$

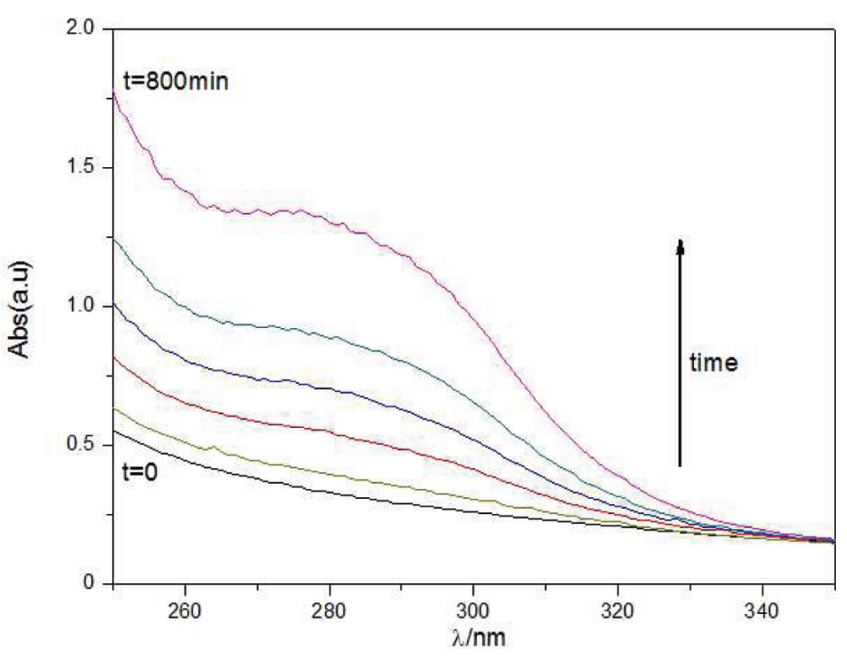

Figure 8. Time evolution of the TAM spectrum during the solubilization process in the presence of $1.4 \mathrm{~g} \mathrm{dm}^{-3}$ of PHEA$\mathrm{PEG}_{5000}-\mathrm{C}_{16} ; \mathrm{TAM}=4 \times 10^{-4} \mathrm{~g}, t=25.0^{\circ} \mathrm{C}$.

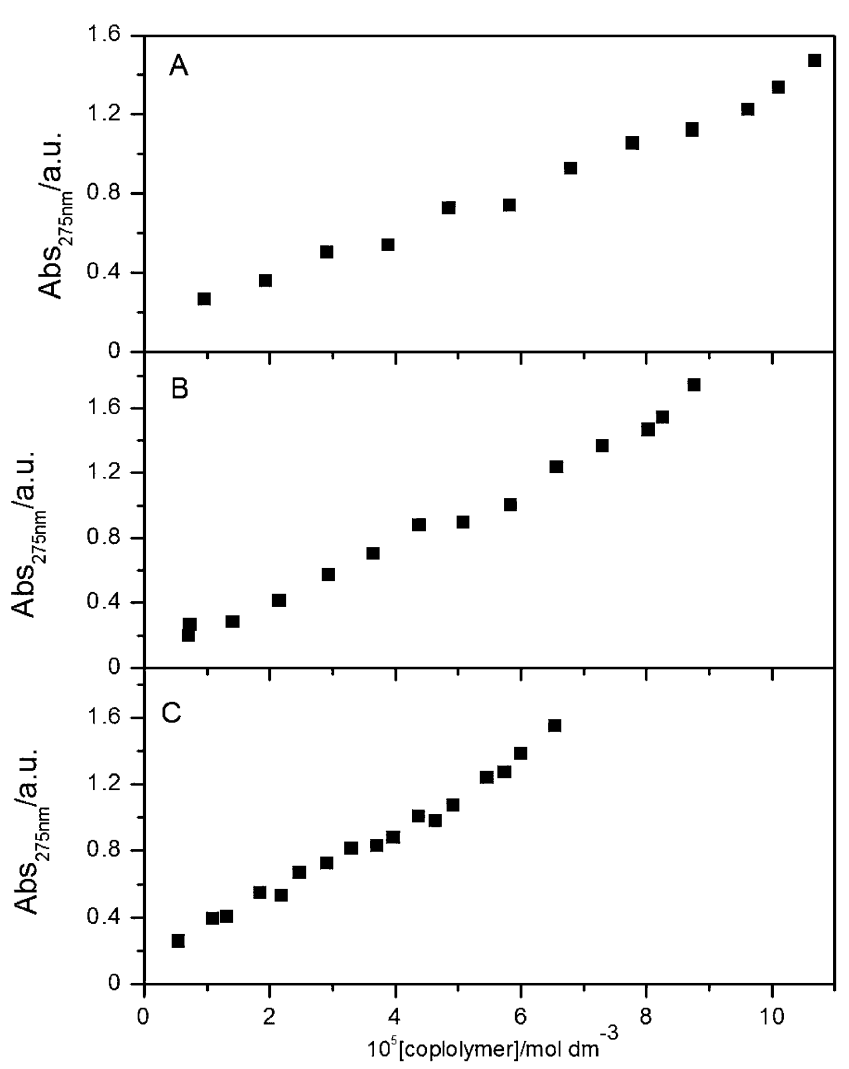

Figure 9. Plot of the final absorbance values of TAM as a function of PHEA-C ${ }_{16}$ (A), PHEA-PEG ${ }_{2000}-\mathrm{C}_{16}$ (B), and PHEA-PEG ${ }_{5000}-\mathrm{C}_{16}(\mathrm{C})$ concentrations; TAM $=4 \times 10^{-4} \mathrm{~g}, t=25.0^{\circ} \mathrm{C}$.

of the sparingly water soluble molecules to the self-aggregate systems according to the following equations

$$
\begin{aligned}
& S_{\mathrm{T}}-S_{\mathrm{w}}=\chi C \\
& K_{\mathrm{B}}=\frac{[\mathrm{TAM}]_{\mathrm{M}}}{[\mathrm{TAM}]_{\mathrm{w}} C}
\end{aligned}
$$

where $S_{\mathrm{T}}$ is the total drug solubility in aqueous surfactant solution, $S_{\mathrm{w}}$ is the drug solubility in water, and $C$ is the concentration of polymeric micelles $\left(C_{\text {total }}-\right.$ cac $)$. 
Since, as we have discussed in section 3.1a, the molar adsorption coefficient value of the TAM in the presence of the polymeric micelles depends on the solubilization sites, i.e., the hydrophilic corona and the hydrophobic core of the micelles, the classical treatment of the equilibrium data is not feasible. Thus, analogously to the kinetic data treatment, we have reasonably considered the polymeric micellar solution as constituted by three pseudophases; namely, the aqueous pseudophase, the hydrophilic corona, and the hydrophobic core and the TAM are partitioned between the pseudophases according to the following equilibria:

$$
\begin{aligned}
& \mathrm{TAM}_{\mathrm{w}} \rightleftarrows \mathrm{TAM}_{\mathrm{p}} \\
& \mathrm{TAM}_{\mathrm{p}} \rightleftarrows \mathrm{TAM}_{\mathrm{c}}
\end{aligned}
$$

with the binding constant given by

$$
\begin{aligned}
K_{\mathrm{B} 1} & =\frac{[\mathrm{TAM}]_{\mathrm{p}}}{[\mathrm{TAM}]_{\mathrm{w}} C} \\
K_{\mathrm{B} 2} & =\frac{[\mathrm{TAM}]_{\mathrm{c}}}{[\mathrm{TAM}]_{\mathrm{p}} C}=\frac{[\mathrm{TAM}]_{\mathrm{c}}}{K_{\mathrm{B} 1}[\mathrm{TAM}]_{\mathrm{w}} C^{2}}
\end{aligned}
$$

This way, the equilibrium absorbance value is the sum of three contributions:

$$
\mathrm{Abs}=\mathrm{Abs}_{\mathrm{w}}+\mathrm{Abs}_{\mathrm{p}}+\mathrm{Abs}_{\mathrm{c}}
$$

where $A b s_{w}, A b s_{p}$, and $A b s_{c}$ are the absorbances related to the TAM solubilized in water, the hydrophilic corona, and the hydrophobic core, respectively.

The absorbance in each pseudophase can be converted into concentration, and eq 9 becomes

$$
\mathrm{Abs}=\varepsilon_{\mathrm{w}}[\mathrm{TAM}]_{\mathrm{w}}+\varepsilon_{\mathrm{p}}[\mathrm{TAM}]_{\mathrm{p}}+\varepsilon_{\mathrm{c}}[\mathrm{TAM}]_{\mathrm{c}}
$$

By dividing each member of eq 10 for $[\mathrm{TAM}]_{\mathrm{w}}$ (which corresponds to the solubility of TAM in pure water) and substituting eqs 7 and 8 we obtain

$$
\frac{\mathrm{Abs}}{[\mathrm{TAM}]_{\mathrm{w}}}=\varepsilon_{\mathrm{w}}+\varepsilon_{\mathrm{w}} K_{\mathrm{B} 1} \mathrm{C}+\varepsilon_{\mathrm{c}} K_{\mathrm{B} 1} K_{\mathrm{B} 2} C^{2}
$$

According to eq 11 , the plot of $A /[\mathrm{TAM}]_{\mathrm{w}}$ as a function of the polymeric micelles concentration has to show a parabolic trend (see Figure 10). Indeed, for the three copolymers used, the nonlinear least-squares analysis of the equilibrium data to eq 11 yields the binding constant values. The results are collected in Table 2 .

Support for the proposed partition scheme has been further provided by the equilibrium data obtained for the copolymer PHEA-PEG $_{2000}$ and PHEA-PEG 5000 .

For these copolymers eq 9 reduces to

$$
\mathrm{Abs}=\mathrm{Abs}_{\mathrm{w}}+\mathrm{Abs}_{\mathrm{p}}
$$

Analogously to the treatment of the above absorbance data, we obtain

$$
\frac{\mathrm{Abs}}{[\mathrm{TAM}]_{\mathrm{w}}}=\varepsilon_{\mathrm{w}}+\varepsilon_{\mathrm{w}} K_{\mathrm{B} 1} \mathrm{C}
$$

The obtained linear trend of the $\mathrm{A} /[\mathrm{TAM}]_{\mathrm{w}}$ as a function of the polymer concentration (see Figure 11) validates the proposed mechanism. The linear least-squares analysis of the data yields the binding constant values of the TAM to the copolymer random coils, whose values are collected in Table 2 .

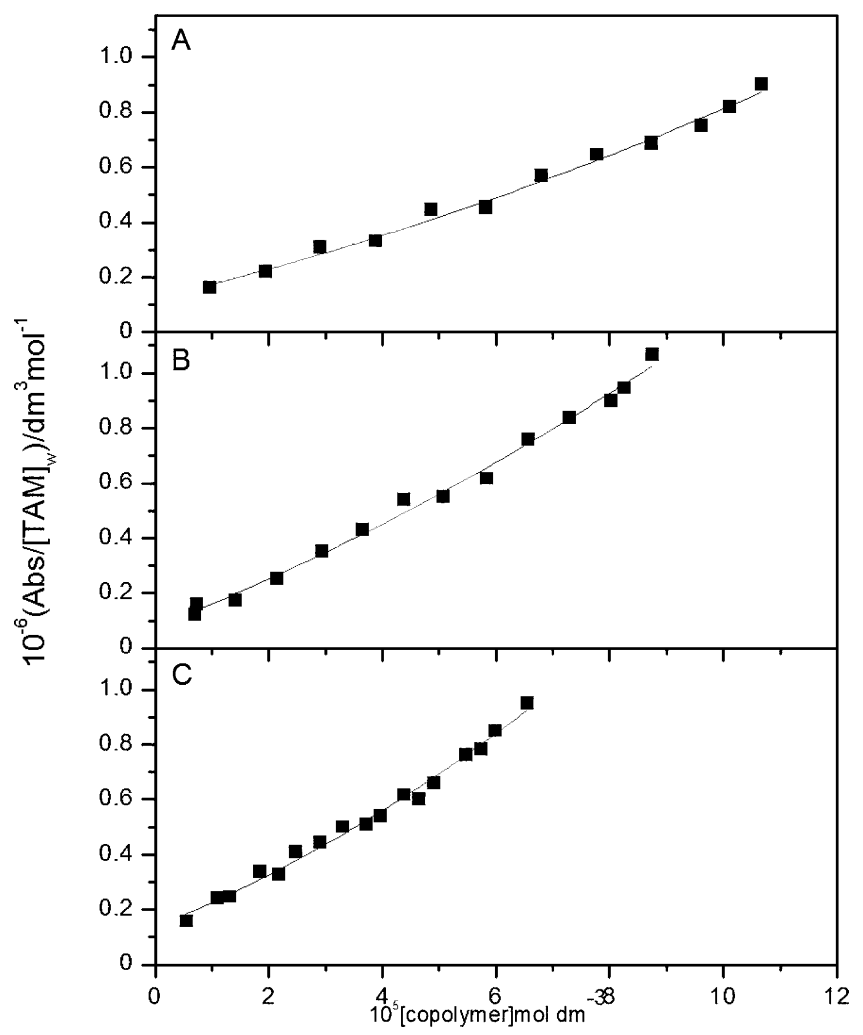

Figure 10. Plot of the ratio $A /[\mathrm{TAM}]_{\mathrm{w}}$ as a function of PHEA-C $\mathrm{C}_{16}$ (A), PHEA-PEG ${ }_{2000}-\mathrm{C}_{16}$ (B), and PHEA-PEG ${ }_{5000}-\mathrm{C}_{16}$ (C) concentrations; TAM $=4 \times 10^{-4} \mathrm{~g}, t=25.0^{\circ} \mathrm{C}$.

It is worth saying that the $\varepsilon_{\mathrm{w}}$ obtained from the analysis is in good agreement with that experimentally determined.

It is possible to notice that the binding constant $K_{\mathrm{B} 1}$ slightly depends on the corona thickness; i.e., the copolymer having a bigger hydrophilic headgroup shows a higher affinity toward the TAM drug, while the $K_{\mathrm{B} 2}$ values, within the experimental error, are almost independent of the copolymer type, as it has been expected if allowance is made that the copolymers have the same hydrophobic tail length. Moreover, it is worth saying that the binding constants of TAM to the PHEA-PEG copolymers are, within the experimental error, in good agreement with those obtained with the PHEA-PEG group belonging to the polymeric micelles.

These results are a further proof of the appropriateness of the proposed solubilization mechanism.

\section{CONCLUSIONS}

The solubilization process of the TAM drug into poly(aspartamide)-based copolymers aqueous solutions has been investigated by means of kinetic and equilibrium studies.

Since the attempt to apply the classical method to the treatment of the obtained data proved not feasible, a novel approach has been proposed, based on the determination of the molar adsorption coefficient of TAM in the polymeric micelles solution and on the consequent individuation of the solubilization sites of TAM into different regions of the polymeric micelles, namely the hydrophilic corona and the hydrophobic core.

Analysis of the kinetic data allowed to propose a multistep solubilization mechanism and to estimate the kinetic constants associated with each step. It has been found that, for all 
Table 2. Binding Constant Values Obtained from the Nonlinear Least-Squares Analysis of the Equilibrium Data to Eq 11

\begin{tabular}{ccccc} 
& PHEA-C $_{16}$ & PHEA-PEG $_{2000}-\mathrm{C}_{16}$ & PHEA-PEG $_{5000}-\mathrm{C}_{16}$ & PHEA-PEG $_{2000}$ \\
$K_{\mathrm{B} 1} / \mathrm{dm}^{3} \mathrm{~mol}^{-1}$ & $(4.1 \pm 0.4) \times 10^{4}$ & $(6.0 \pm 0.4) \times 10^{4}$ & $(7.4 \pm 0.6) \times 10^{4}$ & $(7.1 \pm 0.3) \times 10^{4}$ \\
$K_{\mathrm{B} 2} / \mathrm{dm}^{3} \mathrm{~mol}^{-1}$ & $(7.8 \pm 0.5) \times 10^{4}$ & $(8.5 \pm 0.4) \times 10^{4}$ & $(8.2 \pm 0.6) \times 10^{4}$ & $(8.5 \pm 0.4) \times 10^{4}$ \\
\hline
\end{tabular}

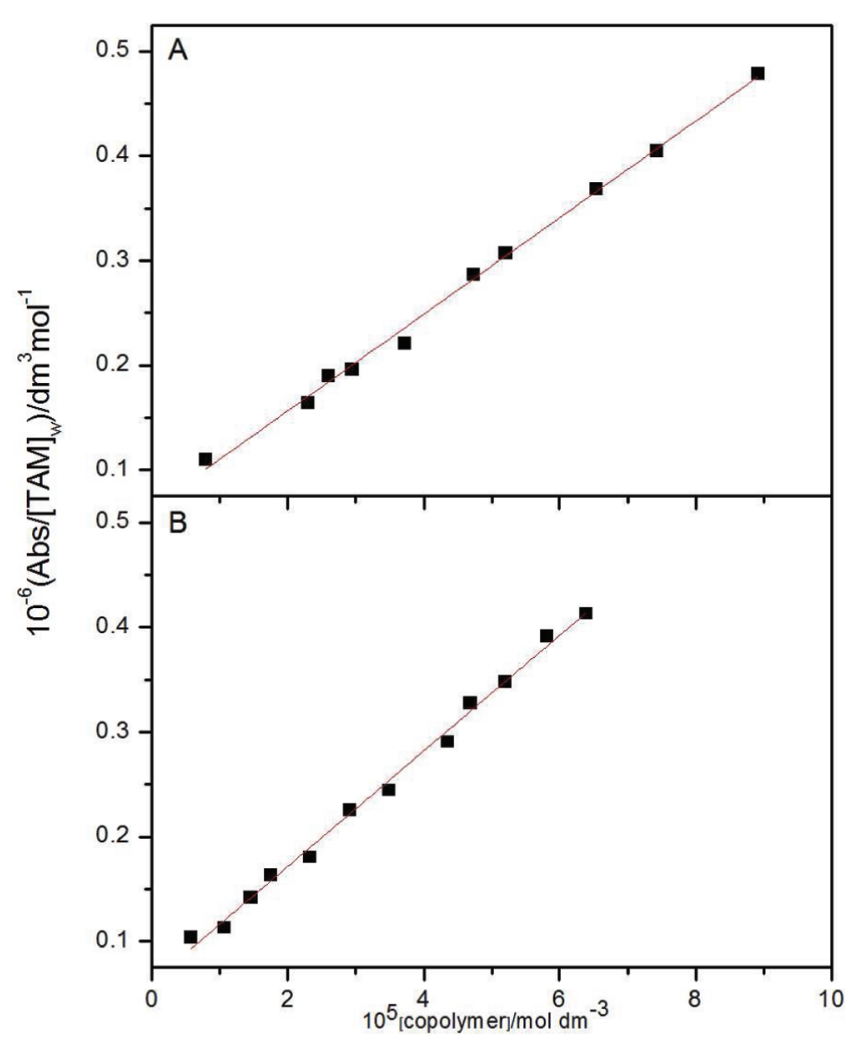

Figure 11. Plot of the ratio $A /[T A M]_{w}$ as a function of PHEA-PEG ${ }_{2000}$ (A) and PHEA-PEG 5000 (B); TAM $=4 \times 10^{-4} \mathrm{~g}, t=25.0^{\circ} \mathrm{C}$.

copolymers used, the solubilization rate constant value of TAM in pure water is always higher than those obtained in the presence of biopolymers, which are independent of the copolymer concentration.

The effect of the thickness of the hydrophilic corona of the copolymers on the kinetic constant values has been investigated, suggesting that the hydrophilic corona represents a barrier which slows down the solubilization into the core.

As for the equilibrium data, they have been treated in a nonconventional way and the partition of the TAM drug between the aqueous pseudophase, the hydrophilic corona, and the hydrophobic core has been suggested and the binding constant of TAM to the hydrophilic corona $\left(K_{\mathrm{B} 1}\right)$ and to the hydrophobic core $\left(K_{\mathrm{B} 2}\right)$ has been determined. It is possible to notice that the binding constant $K_{\mathrm{B} 1}$ slightly depends on the corona thickness; i.e., the copolymer having a bigger hydrophilic headgroup shows a higher affinity toward the TAM drug. This result can be explained in the light of the kinetic results discussed above. In fact, the hydrophilic corona represents a steric barrier which hampers to a certain extent, depending on the thickness, the cross trough itself. Moreover, the KB2 values, within the experimental error, are almost independent of the copolymer type, as it has been expected if allowance is made that the copolymers have the same hydrophobic tail length.

The data reported in this study provided novel and useful information for understanding the processes of drug solubiliza- tion and for the application of polymeric micelles as drug delivery systems of sparingly soluble substances.

\section{AUTHOR INFORMATION}

\section{Corresponding Author}

*E-mail: marialiria.turcoliveri@unipa.it.

\section{Notes}

The authors declare no competing financial interest.

\section{ACKNOWLEDGMENTS}

The authors thank the MIUR for financial support (research funds ex quota $60 \%$ )

\section{REFERENCES}

(1) Kwon, G. S. Crit. Rev. Ther. Drug Carrier Syst. 2003, 20, 357-403.

(2) Bawarski, W. E.; Chidlowsky, E.; Bharali, D. J.; Mousa, S. A. Nanomed.: Nanotechnol. Biol. Med. 2008, 4, 273-282.

(3) Torchilin, V. P. Pharm. Res. 2007, 24, 2333-2334.

(4) Lin, J.; Zhand, S.; Chen, T.; Lin, S.; Jin, H. Int. J. Pharm. 2007, $336,49-57$.

(5) Alvarez-Lorenzo, C; Concheiro, A. J. Drug Delivery Sci. Technol. 2010, 20, 249-257.

(6) Giammona, G.; Carlisi, G.; Palazzo, S. J. Polym. Sci., Polym. Chem. 1987, 25, 2813-2818.

(7) Caliceti, P.; Quarta, S. M.; Veronese, F. M.; Cavallaro, G.; Pedone, E.; Giammona, G. Biochem. Biophys. Acta 2001, 1528, 177186.

(8) Mendichi, R.; Giacometti Schieroni, A.; Cavallaro, G.; Licciardi, M.; Giammona, G. Polymer 2003, 44, 4871-4879.

(9) Cavallaro, G.; Licciardi, M.; Giammona, G.; Caliceti, P.; Semenzato, A.; Salmaso, S. J. Controlled Release 2003, 89, 285-295.

(10) Cavallaro, G.; Maniscalco, L.; Licciardi, M.; Giammona, G. Macromol. Biosci. 2004, 4, 1028-1038.

(11) Civiale, C.; Licciardi, M.; Cavallaro, G.; Giammona, G.; Mazzone, M. G. Int. J. Pharm. 2009, 378, 177-186.

(12) Elsabahy, M.; Perron, M.; Bertrand, N.; Yu, G.; Leroux, J. Biomacromolecules 2007, 8, 2250-2257.

(13) Dar, A. A.; Rather, G. M.; Das, A. R. J. Phys. Chem. B 2007, 111, 3122-3132.

(14) Bath, P. A.; Dar, A. A.; Rather, G. M. J. Chem. Eng. Data 2008, 53, 1271-1277.

(15) Bath, P. A.; Rather, G. M.; Dar, A. A. J. Phys. Chem. 2009, 113, 997-1006.

(16) Saveyn, P.; Cocquyt, E.; Zhu, W.; Sinnaeve, D.; Haustraete, K.; Martins, J. C.; Van der Meeren, P. Phys. Chem. Chem. Phys. 2009, 11, 5462-5468.

(17) Ishii, S.; Ishikawa, S.; Mizuno, N.; Matsuoka, K.; Honda, C.; Endo, K. J. Colloid Interface Sci. 2008, 317, 115-120.

(18) Barreiro-Iglesias, R.; Bromberg, L.; Temchenko, M.; Hatton, T. A.; Concheiro, A.; Alvarez-Lorenzo, C. J.Controlled Release 2004, 97, $537-549$.

(19) Oliveira, C. P.; Vasconcellos, L. C.; Ribeiro, M. E.; Ricardo, N. M.; Souza, T. V.; Costa, F. de M. L. L.; Chaibundit, C.; Yeates, S. G.; Attwood, D. Int. J. Pharm. 2011, 409, 206-208.

(20) Cavallaro, G.; Giammona, G.; Lombardo, R.; Sciascia, L.; Turco Liveri, M. L. Colloids Surf. A Phys. Eng. Aspects 2006, 289, 10-16.

(21) Merli, M.; Sciascia, L.; Turco Liveri, M. L. Int. J. Chem. Kinet. 2010, 42, 587-607.

(22) Salamoun, J.; Macka, M.; Nechvátal, M.; Matousek, M.; Knesel, L. J. Chromatogr. A 1990, 514, 179-187.

(23) Xiarchos, I.; Doulia, D. J. Hazard. Mater. 2006, 136, 882-888. 
(24) Aparna, S.; Gawade, A. K.; Vanjara, M. R. S. Sep. Purif. Technol. 2005, 41, 65-71.

(25) Mall, S.; Buckton, G.; Rawlins, D. A. Int. J. Pharm. 1996, 131, $41-46$. 